\title{
Efeitos da distância entre iscas nas estimativas de abundância e riqueza de formigas em uma floresta de terra-firme na Amazônia Central
}

\author{
Fabricio Beggiato BACCAROํㅗㄴ Suzana Maria KETELHUT², José Wellington de MORAIS³
}

\begin{abstract}
RESUMO
Coletas com iscas são amplamente utilizadas para investigar a atividade de formigas de solo e vegetação e também podem ser empregadas para estimar o número de espécies e a abundância de formigas. Apesar de ser barata e fácil de implementar, a riqueza e abundância das formigas estimadas por iscas podem ser enviesadas por alguns fatores, como a distância entre iscas. Neste trabalho, avaliamos se a distância entre iscas altera as estimativas de abundância e riqueza de formigas e qual distância entre iscas resulta na melhor relaçáo entre custo financeiro e número de espécies amostradas, objetivo da maioria dos relatórios de impacto ambiental. Amostramos 30 transectos de $100 \mathrm{~m}$ com distância entre iscas diferentes $(2,5 ; 3,4 ; 5 ; 6,7 ; 10$ e $20 \mathrm{~m})$, distribuídos em uma área de $1 \mathrm{~km}^{2}$ em uma floresta de terra-firme ao norte de Manaus. Independente da distância entre iscas, o número de espécies coletado a cada cinco iscas, foi aproximadamente 8 , e a abundância média foi maior que 50 indivíduos. No entanto o número de espécies por isca foi maior em transectos com maior distância entre iscas. Transectos com distância entre iscas de 10 e $20 \mathrm{~m}$, coletaram 50\% mais espécies por isca que transectos com iscas distantes a 2,5 e 3,4 m entre si. Nossos resultados sugerem que nesta área, a amostragem mais eficiente desse método de coleta seria distribuir 450 iscas a cada $10 \mathrm{~m}$ ou $20 \mathrm{~m}$ no solo da floresta.
\end{abstract}

PALAVRAS-CHAVE: conservação, curva de acumulação de espécies, esforço de coleta, floresta tropical, invertebrados de solo, relatório de impacto ambiental.

\section{Effects of bait spacing on ant abundance and richness in one forest at Central Amazonia}

\begin{abstract}
Baits are widely used to investigate the activity of terrestrial and arboreal ants, but also can be used to estimate the ant abundance and species richness. Despite the fact that baiting are cheap and easy to implement, many factors, such as the distance between baits, may influence the estimate of ant species richness and abundance. In this study, we evaluated the effects of bait spacing on abundance and the number of ant species. We also demonstrate which distance between baits showed the best relationship between costs and number of ant species sampled. We sampled 30 transects of $100 \mathrm{~m}$ with bait spacing ranging from 2.5 to $20 \mathrm{~m}$, spread over $1 \mathrm{~km}^{2}$ of a "terra-firme" forest situated at North of Manaus. The bait spacing did not affect the ant diversity estimative. Regardless bait spacing, the number of species collected every five baits was around 8 , and the average abundance was approximately 50 individuals. However the number of species per bait was higher in transects with larger bait spacing. Transects with bait spacing of $10 \mathrm{~m}$ and $20 \mathrm{~m}$, collected $50 \%$ more species than transects with baits placed $2.5 \mathrm{~m}$ and 3.4 $\mathrm{m}$ apart. Our results suggest that at this forest, the most efficient sampling design using only baits would be place 450 baits every $10 \mathrm{~m}$ or $20 \mathrm{~m}$ at the forest floor.
\end{abstract}

KEYWORDS: conservation, environmental impact report, sampling effort, soil invertebrates, species accumulation curve, tropical forest.

\footnotetext{
1 Instituto Nacional de Pesquisas da Amazônia. fabricera@gmail.com

2 Instituto Nacional de Pesquisas da Amazônia. s_ketelhut@yahoo.com

${ }^{3}$ Instituto Nacional de Pesquisas da Amazônia. morais@inpa.gov.br
} 


\section{INTRODUÇÃO}

A inclusão de invertebrados terrestres em inventários de biodiversidade e em relatórios de impactos ambientais aumentou rapidamente na última década (Oliver e Beattie, 1996; Ward et al., 2001; New, 2008). Dentre os invertebrados terrestres, as formigas são frequentemente usadas nesses estudos por representarem uma grande porção da diversidade de espécies animais nas florestas tropicais (Erwin, 1989), constituírem uma das maiores proporçôes da biomassa animal (Fittkau e Klinge, 1973; Ellwood e Foster, 2004) e serem localmente dominantes em relação a outros macro artrópodes (Stork, 1991). A eficiência de coleta de um grupo táo diverso e abundante, como das formigas, é um componente chave no desenho de inventários rápidos de biodiversidade ou de relatórios de impactos ambientais que visam atender em tempo hábil certas demandas da sociedade (Oliver e Beattie, 1993; New, 2008).

Coletar formigas com iscas atrativas é um método simples, barato e fácil de implementar. Esse método de coleta é amplamente utilizado para investigar a atividade de formigas que vivem no solo e na vegetação (Savolainen e Vepsäläinen, 1988; Porter e Savignano, 1990; Davidson, 1998; Bestelmeyer et al., 2000; Brandão et al., 2000; Albrecht e Gotelli, 2001), mas também pode ser empregado para estimar o número de espécies de formigas (Benson e Harada, 1988; Silva et al., 2004) ou como método complementar em inventários (Vasconcelos et al., 2009). No entanto a coleta com iscas normalmente amostra de forma tendenciosa a composição da comunidade. Algumas formigas, denominadas dominantes, possuem comportamento agressivo e podem deslocar ou impedir outras formigas de usarem determinado recurso ou visitar uma isca (Vepsäläinen e Savolainen, 1990; Andersen, 1997; Holway et al., 2002). Dessa forma, indivíduos que forrageiam solitariamente ou espécies com comportamento subordinado podem ser subestimadas (Bestelmeyer et al., 2000). Interaçôes antagônicas como essa, podem inserir um viés nas comparaçôes entre tratamentos de um estudo ou entre comparaçôes faunísticas que utilizam somente coletas com iscas.

Muitos trabalhos investigaram os efeitos do tipo de iscas (Davidson, 1997; Feener Jr. e Schupp, 1998; Bestelmeyer $e t$ al., 2000; Yanoviak e Kaspari, 2000; Kaspari e Yanoviak, 2001) ou de variaçôes ambientais (Cerdá et al., 1998; Bestelmeyer, 2000; Hahn e Wheeler, 2002; Silva et al., 2004) na resposta comportamental e, consequentemente, na eficiência de amostragem das espécies. No entanto, a distância entre iscas também pode afetar o forrageamento das formigas e alterar os resultados da amostragem. Mantendo-se o tamanho da área amostral, reduzir a distância entre iscas significa aumentar sua concentração no espaço. Isto poderia afetar o número de espécies e indivíduos detectados pela amostragem de duas maneiras diferentes. Iscas mais próximas podem aumentar a chance de amostrar mais espécies de formigas por concentrar o efeito atrativo das iscas ou simplesmente amostrar em maior detalhe (mais iscas por unidade de espaço) o folhiço/ solo da floresta. Por outro lado, em transectos com menor distância entre iscas, mais iscas seriam oferecidas dentro da área de forrageio das colônias de espécies dominantes e a pressão competitiva exercida por essas espécies sobre espécies subordinadas poderia resultar em um número menor de espécies amostradas. O objetivo deste trabalho foi de determinar se a distância entre iscas afeta a abundância e o número de espécies de formigas coletadas e qual densidade de iscas maximiza a relação entre número de espécies amostrado por esforço de coleta.

\section{MÉTODOS}

\section{Área de estudo}

Este estudo foi conduzido em um sítio de pesquisa do projeto TEAM (Tropical Ecology, Assessment e Monitoring Initiative), situado na reserva 3402 (Cabo Frio) do Projeto Dinâmica Biológica de Fragmentos Florestais (PDBFF) $\left(59^{\circ} 54^{\prime} 59^{\prime \prime} \mathrm{O} ; 2^{\circ} 23^{\prime} 37^{\prime \prime} \mathrm{S}\right)$, a aproximadamente $80 \mathrm{~km}$ ao Norte de Manaus. O local de coleta é composto por uma grade de trilhas de $1 \mathrm{~km}$ regularmente espaçadas a cada 200 $\mathrm{m}$, cobrindo uma área de $1 \mathrm{~km}^{2}$ de floresta de terra-firme pouco perturbada. $\mathrm{O}$ terreno é formado basicamente por platôs com altitudes variando de 50 a $120 \mathrm{~m}$ em relaçáo ao nível do mar, com predominância de solos argilosos nos platôs e arenosos nos baixios. Grande parte desses baixios possuem igarapés durante toda a estaçáo seca (Lovejoy et al., 1991). A flora é extremamente diversificada, com aproximadamente 1.000 espécies de árvores com altura entre 30 e 35 metros, e com árvores emergentes podendo alcançar 50 metros de altura (Lovejoy et al., 1991).

A região de Manaus é caracterizada por um clima tropical úmido (Holdridge, 1967) com precipitação média anual em torno de $2.1 \mathrm{~m}$. A estaçáo chuvosa ocorre de novembro a maio e a estação seca de junho a outubro, sendo o mês de setembro normalmente o mês de menor precipitação (Ribeiro e Adis, 1984). A temperatura média anual é de $26^{\circ} \mathrm{C}$ existindo pouca variaçáo térmica durante o ano, com as temperaturas médias mensais diferindo entre si em menos que $3{ }^{\circ} \mathrm{C}$ (Salati, 1985). A maior variação de temperatura ocorre ao longo do dia, podendo chegar a $8^{\circ} \mathrm{C}$.

\section{Delineamento amostral}

Amostramos 30 transectos de $100 \mathrm{~m}$ entre os meses de março e outubro de 2005, utilizando seis distâncias entre iscas como tratamentos (de 2,5 m a 20 m de distância entre as iscas; Tabela 1). O tamanho dos transectos e as classes de distância entre as iscas foram escolhidos por serem frequentemente 
empregados em inventários com iscas (Benson e Harada, 1988; Belstelmeyer, 2000; Silva et al., 2004; Vasconcelos et al., 2004; Achury et al., 2008). Os transectos foram distribuídos dentro da grade de coleta de $1 \mathrm{~km}^{2}$, com uma distância mínima de 200 $m$ entre si. A seqüência de coleta dos transectos foi sorteada para evitar que transectos com a mesma distância entre iscas fossem amostrados sempre no mesmo horário, diminuindo possíveis efeitos decorrentes da variação diária da atividade das formigas (Bestelmeyer, 2000). Para minimizar o efeito da topografia sobre a comunidade de formigas (Vasconcelos et al., 2003) os transectos foram dispostos em áreas planas.

Oferecemos em cada ponto de coleta aproximadamente $5 \mathrm{~g}$ de isca composta da mistura de sardinha enlatada (600 g) e goiabada $(500 \mathrm{~g})$, sobre pedaços de cartáo plástico com dimensóes de 10 por $7 \mathrm{~cm}$. Após 60 minutos, a abundância de todas as espécies de formigas presentes nas iscas foi registrada de acordo com a escala de abundância proposta por Andersen (1997): 1, 1 formiga; 2, 2-5 formigas; 3, 6-10 formigas; 4, 11-20 formigas; 5, 21-50 formigas; 6, > 50 formigas. Entre 1 a 5 indivíduos de cada espécie de formiga que estavam sobre o cartão, foram coletados e conservados em álcool 70\% para identificação em laboratório. Os espécimes foram identificados e depositados na Coleção Entomológica do Instituto Nacional de Pesquisas da Amazônia - INPA.

Tabela 1 - Número de transectos, número de iscas e distância entre as iscas de transectos de $100 \mathrm{~m}$ distribuídos no sítio de pesquisa do Cabo Frio, Manaus.

\begin{tabular}{lcc}
\hline $\begin{array}{l}\text { Número de } \\
\text { transectos }\end{array}$ & $\begin{array}{c}\text { Número de iscas por } \\
\text { transecto }\end{array}$ & $\begin{array}{c}\text { Distância entre iscas } \\
(\mathrm{m})\end{array}$ \\
\hline 5 & 41 & 2,5 \\
5 & 31 & 3,3 \\
5 & 21 & 5,0 \\
5 & 16 & 6,7 \\
5 & 11 & 10,0 \\
5 & 6 & 20,0 \\
\hline
\end{tabular}

\section{Análise dos dados}

Para controlar o efeito do esforço amostral diferente (o número de iscas por transecto variou de 6 a 41) utilizamos um método análogo à rarefação de amostras. Para cada transecto, calculamos a média do número de espécies amostrado em 100 sorteios de cinco iscas. Dessa forma, todos os transectos ficaram com o mesmo número de subamostras ao longo das análises comparativas, sendo possível comparar a riqueza e abundância entre os transectos e avaliar o efeito da distância entre iscas sobre a amostragem da comunidade. Os efeitos da distância entre iscas sobre o número de espécies e a abundância das formigas estimadas pelos sorteios (a partir daqui denominados de número relativo de espécies e abundância relativa, respectivamente) foram investigados separadamente por Análises de Variância (ANOVA).

Os possíveis efeitos da distância entre iscas sobre o número de espécies coletado por isca e sobre a abundância das espécies por isca, que são estimativas da eficiência do método de coleta, também foram investigados por Análises de Variância (ANOVA). Como um dos objetivos deste estudo é determinar qual esforço de coleta apresenta a melhor relaçáo entre recurso financeiro e número de espécies amostrado, comparamos o número de espécies coletado por isca entre os tratamentos e corrigimos o nível de significância decorrente de comparações múltiplas pelo método de Bonferroni. Usamos análises de resíduos para determinar se a distribuiçáo dos resíduos e os modelos utilizados atenderam às premissas das análises.

Construímos curvas do coletor por tratamento e de todas as amostras em conjunto para detalhar possíveis efeitos da distância entre iscas na amostragem de espécies de formigas. A curva do coletor estima através do processo de rarefaçáo das amostras (ou indivíduos) o número de espécies esperado para subamostras aleatórias do conjunto dos dados. Calculamos o número de espécies estimado (usando os índices Chao 1, Jackknife 1 e Bootstrap) para indicar um esforço de coleta mínimo para área de estudo (Colwell e Coddington 1994). Para fornecer uma visão mais abrangente da eficiência de coleta com iscas, comparamos nossos resultados com outros trabalhos que utilizaram esse método de coleta em ambientes florestados na Amazônia. Usamos o programa estatístico R (The R Project for Statistical Computing) em todas as análises e gráficos.

\section{RESULTADOS}

Ao total, 112 espécies/morfoespécies de formigas foram coletadas, com média de 20,1 espécies de formigas por transecto. $\mathrm{O}$ número máximo de espécies coletadas em uma isca foi cinco com média de 1,97 espécies por isca. Apenas 22 iscas (menos de 4\%) não foram visitadas por formigas durante o estudo e cerca de $13 \%$ das iscas tinham somente uma espécie ao final do tempo de coleta. Como em outras regiōes da Amazônia o gênero Pheidole foi o mais abundante (1.082 ocorrências) e representou quase $40 \%$ do número de espécies coletadas. Crematogaster tenuicula Forel foi a espécie mais amostrada, sendo encontrada em $25,9 \%$ das iscas oferecidas.

$\mathrm{O}$ número relativo de espécies e a abundância relativa das formigas foram similares entre os tratamentos (ANOVA, $F_{5,24}=1,601 ; P=0,198$ e $F_{5,24}=0,723 ; P=0,613$, respectivamente). Em média, o número de espécies coletado com cinco iscas, independente da distância entre as iscas, foi de aproximadamente 8 , e a abundância das formigas, estimada pela escala de abundância em cinco iscas por transecto, foi de 35,1 (que corresponde a aproximadamente 300 indivíduos; Figura 1). 

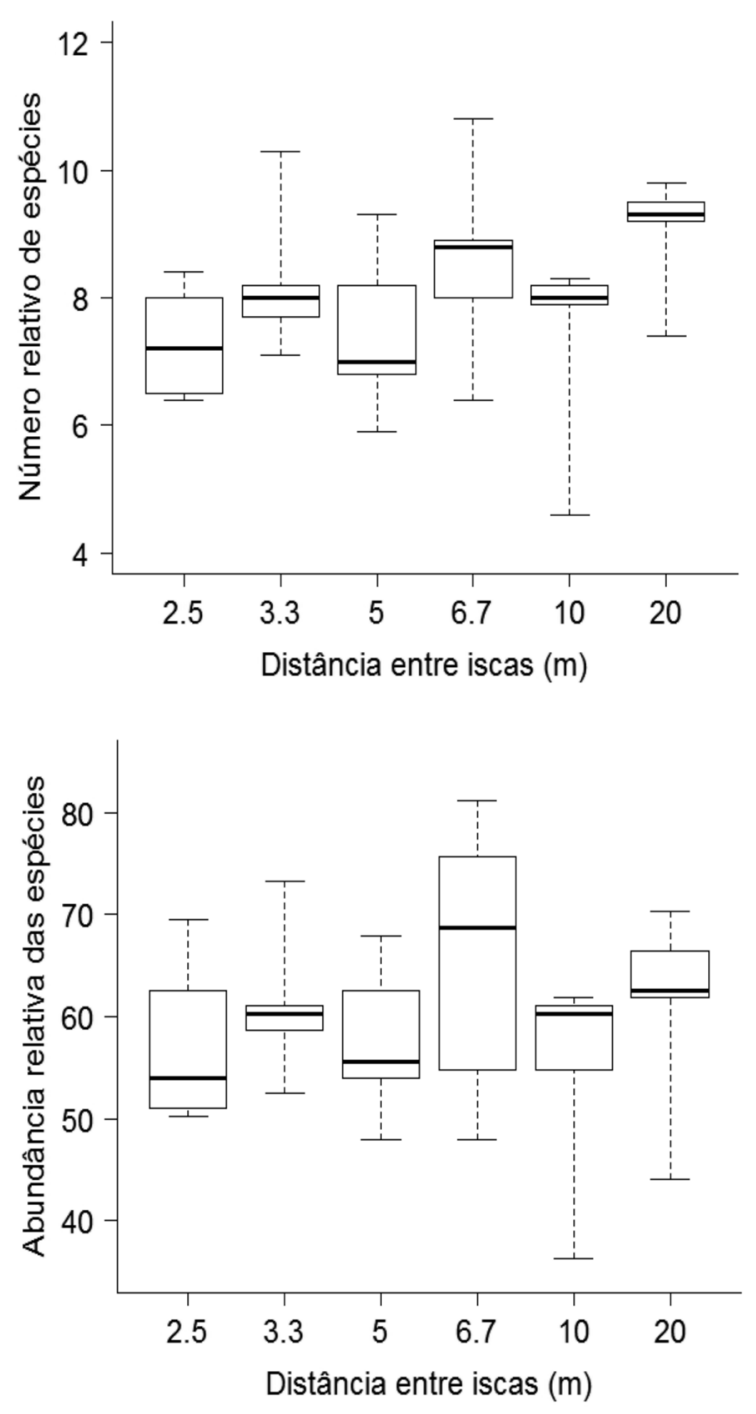

Figura 1 - Número relativo de espécies e abundância relativa de formigas em cinco iscas em transectos com distância entre iscas variando de $2,5 \mathrm{~m}$ a 20 $\mathrm{m}$, amostrados no sítio de pesquisa do Cabo Frio, Manaus. A linha horizontal mais grossa representa a mediana, a caixa 0 intervalo interquatil e as linhas tracejadas os valores extremos.

A abundância das formigas por isca foi similar nos transectos com distância entre iscas diferentes (ANOVA, $F_{5,24}$ $=0,253 ; P=0,934)$. Independentemente da distância entre iscas, a abundância média foi de 6,81 da escala de abundância, ou seja, mais de 50 indivíduos por isca (Figura 2). No entanto a relação entre número de espécies por esforço de coleta variou entre os tratamentos. O número de espécies coletado por isca foi maior nos transectos com maior distância entre iscas (ANOVA, $\left.F_{5,24}=5,053 ; P=0,002\right)$. Transectos com iscas distantes $10 \mathrm{~m}$ e $20 \mathrm{~m}$ entre si, coletaram aproximadamente $50 \%$ mais espécies por isca que transectos com iscas distantes a 2,5 m e 3,3 m entre si (Figura 2). Apesar disso, o número acumulado de espécies por tratamento foi similar (Figura 3a). Como as coletas foram realizadas em áreas de mata contínua, a curva do coletor total não mostrou tendência à estabilização (Figura 3b). O número de espécies estimado variou de 127 a 140 espécies (Chao $1=137,3 \pm 11,73$; Jackknife $1=140,0$ $\pm 4,99$ e Bootstrap $=127,0 \pm 2.89$ ), indicando que entre $10 \mathrm{e}$ $21 \%$ das espécies não foram amostradas.
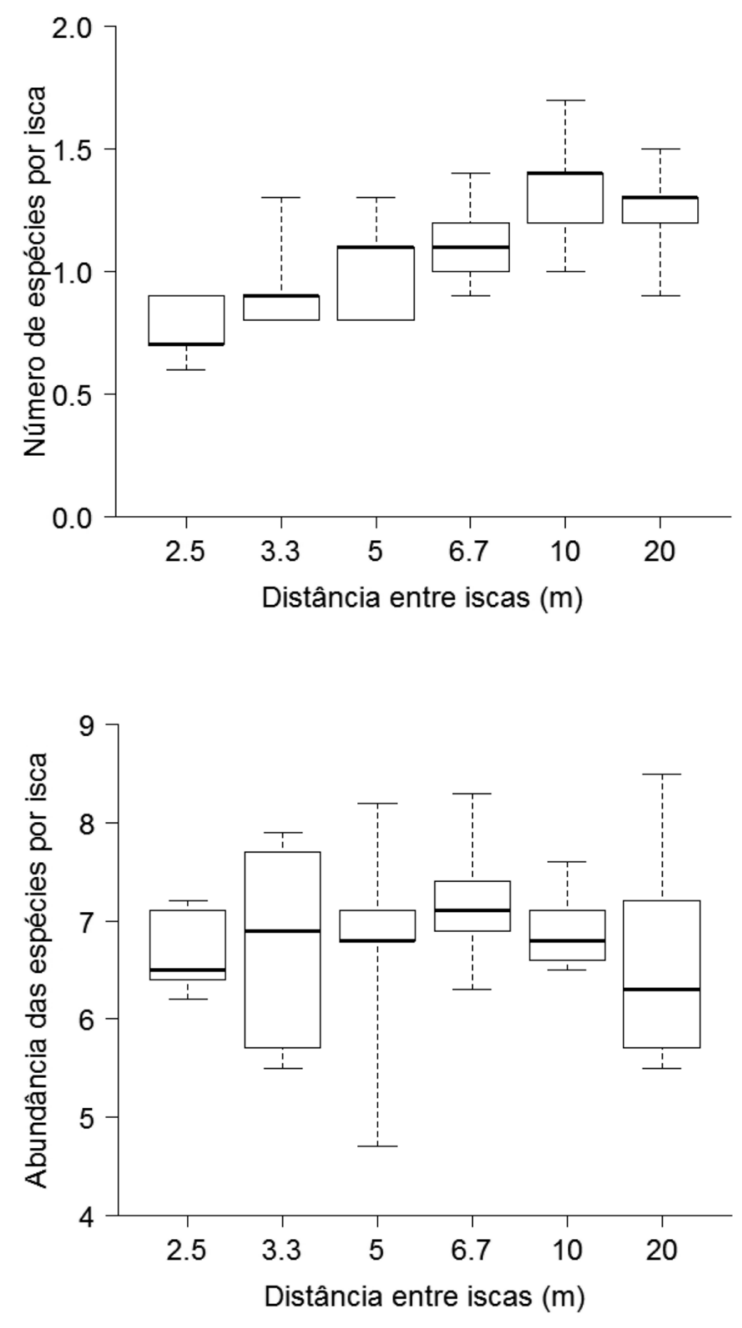

Figura 2 - Número de espécies e abundância de formigas por isca em transectos com distância entre iscas variando de $2,5 \mathrm{~m}$ a $20 \mathrm{~m}$, amostrados no sítio de pesquisa do Cabo Frio, Manaus. A linha horizontal mais grossa representa a mediana, a caixa o intervalo interquatil e as linhas tracejadas os valores extremos. 

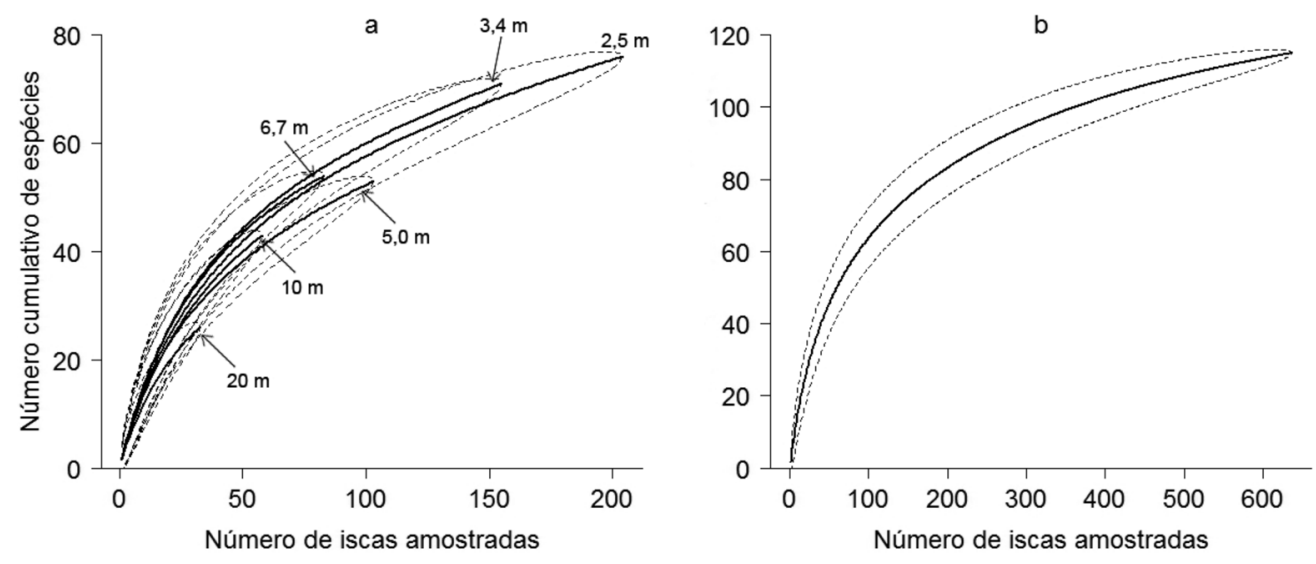

Figura 3 - Curvas do coletor (rarefação) por tratamento (a) e de todos os transectos combinados (b). As linhas tracejadas representam o intervalo de confiança de $95 \%$ ao redor dos valores estimados.

\section{DISCUSSÃO}

A distância entre iscas não afetou o número de espécies coletado nas comparaçóes com esforço de coleta padronizado. Independentemente da distância entre iscas, cinco iscas oferecidas sobre o folhiço dessa floresta atraíram em média 8 espécies. $\mathrm{O}$ incremento de novas espécies também foi similar entre os tratamentos, sugerindo que a quantidade de iscas oferecidas, que variou em uma ordem de grandeza, amostrou de forma similar a fauna do local. No entanto, os transectos com iscas mais concentradas, ou seja, os transectos com menor distância entre iscas atraíram proporcionalmente menos espécies por isca. As colônias de formigas forrageiam mais intensamente sobre recursos mais próximos e abundantes (Bernstein, 1979; Sanders e Gordon, 2002) e principalmente nos transectos com iscas mais concentradas, a maior intensidade de forrageio e eventual monopolio ou dominância poderia impedir o acesso de algumas espécies ao recurso oferecido. No entanto, proporcionalmente mais iscas foram controladas por espécies dominantes nos transectos com maior distância entre iscas, sugerindo que existe uma demanda conflitante entre a resposta comportamental e a densidade de recursos (ver Baccaro et al., 2010).

A variação no tamanho da área de forrageio das colônias pode explicar porque coletamos mais espécies por isca nos transectos com iscas mais distantes. Nas florestas tropicais, o tamanho da área de forrageio das colônias varia muito e normalmente está relacionado ao tamanho das colônias e ao local de nidificação. Por exemplo, formigas que nidificam no folhiço normalmente formam colônias pequenas (10-100 indivíduos) e tem área de forrageio restrita ao redor do ninho (Byrne, 1994). Outras espécies (i.e. C. tenuicula e Wasmannia auropunctata (Roger), coletadas nesse estudo) podem formar "super colônias" ou colônias polidômicas utilizando vários locais de nidificaçáo independentes, aumentando o tamanho da área de forrageio da colônia (Le Brenton et al., 2005; Longino, 2003). Como as espécies que tem o hábito de criar colônias polidômicas, C. tenuicula, C. brasiliensis Weber e W. auropunctata, foram encontradas em $53 \%$ das iscas oferecidas, é provável que nos transectos com menor distância entre iscas, proporcionalmente mais iscas foram oferecidas dentro da área de forrageio dessas espécies. A amostragem de indivíduos da mesma colônia em diversas subamostras por transecto resulta em menor número de espécies coletado por isca nos transectos com maior esforço de coleta, ou seja, nos transectos com menor distância entre iscas.

Animais solitários procuram por recursos disponíveis e a abundância de alguns insetos está positivamente correlacionada com a densidade de recursos (Messina, 1991; Heard, 1998). No entanto, as formigas são organismos sociais e modulares tendo uma relaçấo com meio físico relativamente similar às plantas (Andersen, 1995). As colônias de formigas são relativamente sésseis e não mudam de local em função de recursos efêmeros, como neste caso, as iscas. No entanto, o balanço energético entre a quantidade de energia necessária para a manutenção da colônia e a quantidade de energia consumida na procura de alimento é feito no nível da colônia. Algumas espécies podem regular a intensidade de forrageio em função da quantidade de recursos disponíveis, diminuindo o forrageio em períodos com menor disponibilidade de recursos e forrageando mais intensamente em locais com recursos mais abundantes (Gordon e Kulig, 1996; Sanders e Gordon 2002, 2003). No entanto, nossos resultados sugerem que a densidade de recursos não afeta as estimativas de abundância de formigas. Tanto nas comparaçóes com o esforço de coleta 
padronizado, como nas comparaçóes de abundância de formigas por isca, o número de indivíduos foi similar entre os tratamentos. É possível que a variação na densidade de recursos tenha regulado a intensidade de forrageamento de algumas espécies, mas no nível da comunidade esse efeito foi imperceptível, porque a diminuição da abundância de algumas espécies pode compensar o aumento da abundância de outras espécies. Além disso, o tempo de exposição das iscas pode ter mascarado possíveis variaçóes na quantidade de formigas atraídas. Normalmente em ambientes florestados, as interações competitivas se estabilizam após 60 minutos de exposição das iscas (Davidson, 1998, Andersen, 2000). Dessa forma, as espécies que recrutam em massa, que correspondem a maioria das espécies atraídas pelas iscas, tiveram tempo de concentrar um grande número de indivíduos, frequentemente mais de 50 indivíduos, ou seja valor 6 (máximo) da escala de abundância ao redor da isca.

O número total de espécies amostrado neste trabalho foi similar ao reportado na literatura para outras regiôes florestadas da Amazônia (Tabela 2). A diferença entre o número de espécies amostrado por estudos anteriores (58-122) deve-se provavelmente a variação no esforço de coleta e ao tipo de ambiente florestal amostrado (Tabela 2). Inventários com esforço de coleta concentrado em áreas relativamente pequenas (Vasconcelos et al. 2004; Vasconcelos et al. 2010), amostraram menos espécies que estudos com esforço de coleta distribuídos em áreas maiores (Vasconcelos e Vilhena, 2006; Souza, 2010). Esse resultado é esperado porque áreas maiores normalmente são mais heterogêneas, e ambientes mais heterogêneos frequentemente abrigam mais espécies que áreas menores e mais homogêneas (Báldi 2008).

As formigas são frequentemente descritas como organismos termofílicos (Cerdá et al., 1998; Cerdá et al., 2002). Locais mais secos normalmente comportam menos espécies que locais mais úmidos e locais mais quentes concentram mais espécies por unidade de área que locais mais frios (Dunn et al., 2009; Vasconcelos et al., 2010). É possível que florestas mais secas comportem menos espécies de formigas atraídas por iscas que florestas mais úmidas (Tabela 2). Achury et al. (2008) coletaram em florestas secas da Amazônia colombiana praticamente metade do número de espécies amostrado nas florestas úmidas da Amazônia central brasileira, levando em consideração esforço amostral similar (Benson e Harada, 1988). No entanto, a diferença entre o número de espécies amostrado em florestas secas e úmidas da Amazônia é baseada somente em uma comparação e deve ser melhor investigado.

Embora o desenho amostral deva ser direcionado para atender o objetivo de cada estudo, a inclusáo de invertebrados terrestres em processos de licenciamento ambiental, relatórios de impacto ambiental e em inventários de biodiversidade demanda maior atenção quanto à eficiência na amostragem. Um esforço de coleta mal dimensionado pode coletar muitos invertebrados desnecessariamente, sobrecarregando a equipe de pesquisa no processamento do material coletado e na elaboração do produto final. Por outro lado, se o esforço de coleta for insuficiente o inventário irá subestimar a diversidade de um local e pode levar a conclusóes enganosas. Uma das maneiras de estimar o número de espécies de um local é realizar coletas aleatórias e produzir uma curva de acumulação de espécies (Colwell e Coddington 1994). Teoricamente qualquer amostragem de campo pode atingir a assíntota da curva do coletor, demonstrando que todas as espécies foram amostradas (Gotelli e Colwell, 2001). No entanto, a assíntota ou estabilização da curva normalmente é atingida em táxons com número reduzido de espécies, como por exemplo, jacarés e tartarugas (Rueda-Almonacid et al., 2007). Em táxons megadiversos, como as formigas, conforme mais indivíduos são amostrados, mais espécies são registradas. Isso acontece porque nesses grupos existem muitas espécies raras (Gotelli e Colwell, 2001; Ugland et al., 2003). Apesar da curva do coletor (Figura 3) não apresentar sinais de estabilização, nossos resultados sugerem que para amostrar $80 \%$ das espécies, levando em

Tabela 2 - Local de coleta, área amostrada, número de iscas empregado e número de espécies coletado disponíveis em trabalhos que usaram iscas atrativas para coletar formigas em ambientes florestados na Amazônia.

\begin{tabular}{lcccc}
\hline Local & $\begin{array}{c}\text { Área total amostrada } \\
\text { (ha) }\end{array}$ & $\begin{array}{c}\text { Número de } \\
\text { iscas }\end{array}$ & $\begin{array}{c}\text { Número de } \\
\text { espécies }\end{array}$ & Fonte \\
\hline Bacia do Rio Cauca, Colômbia & 1,59 & 397 & 66 & Achury et al. 2008 \\
Projeto Dinâmica de Fragmentos Florestais, AM & 1,44 & 392 & 111 & Benson e Harada 1988 \\
$\begin{array}{l}\text { Reserva Ducke, AM; Estação Ecológica de Maracá } \\
\text { e Parque Nacional do Viruá, RR }\end{array}$ & 7500.00 & 900 & 122 & Souza 2009 \\
Florestas de Alter do Chão, PA & 127,50 & 680 & 115 & Vasconcelos e Vilhena 2006 \\
\hline Parque Nacional do Jaú, AM & 0,14 & 108 & 58 & Vasconcelos et al. 2004 \\
Ao longo dos Rios Solimões e Amazônas, AM e PA & 2,34 & 1040 & 98 & Vasconcelos et al. 2010 \\
\hline Projeto Dinâmica de Fragmentos Florestais, AM & 100 & 630 & 112 & presente estudo \\
\hline
\end{tabular}


consideração a estimativa mais conservadora (Bootstrap), seria necessário distribuir aproximadamente 450 iscas no solo dessa floresta. Se o objetivo de um estudo for inventariar rapidamente e com menor esforço de coleta o maior número de espécies possível, a amostragem mais eficiente desse método de coleta seria distribuir 450 iscas a cada $10 \mathrm{~m}$ ou $20 \mathrm{~m}$ pelo solo da floresta. Esse desenho amostral seria o mais eficiente na relação custo financeiro/número de espécie amostrado.

\section{AGRADECIMENTOS}

Agradecemos a Rayk William de Oliveira, Marcio Pereira e Vilhena, que nos ajudaram durante o trabalho de campo e identificaçáo das formigas. Durante esse trabalho, F. Baccaro foi bolsista CNPq e contou com apoio financeiro do Projeto TEAM-Manaus. Thiago Izzo, Helder Espírito-Santo, Jorge L. Souza e dois revisores anônimos fizeram comentários importantes nas primeiras versōes desse manuscrito. Esse é o artigo 569 da série técnica do PDBFF/INPA-STRI.

\section{BIBLIOGRAFIA CITADA}

Achury, R.; Ulloa P.C.D.; Arcila, Á.M. 2008. Composición de hormigas e interacciones competitivas con Wasmannia auropunctata en fragmentos de Bosque seco Tropical. Revista Colombiana de Entomología, 34: 209-216.

Albrecht, M.; Gotelli N.J. 2001. Spatial and temporal niche partitioning in grassland ants. Oecologia, 126: 134-141.

Andersen, A.N. 1995. A Classification of Australian Ant Communities, Based on Functional Groups Which Parallel Plant life-forms in relation to stress and disturbance. Journal of Biogeography, 22: 15-29.

Andersen, A.N. 1997. Functional groups and patterns of organization in North American ant communities: a comparison with Australia. Journal of Biogeography, 24: 433-460.

Andersen, A.N. 2000. A global ecology of rainforest ants: functional groups in relation to environmental stress and disturbance, $\mathrm{p}$. 25-34. In: Agosti, D.; Majer, J. D.; Alonso, L. E.; Schultz, T. R. (Eds.). Ants: Standard methods for measuring and monitoring biodiversity. Smithsonian Institution Press, Washington.

Báldi, A. 2008. Habitat heterogeneity overrides the species-area relationship. Journal of Biogeography, 35: 675-681.

Baccaro, F.B.; Ketelhut, S.M.; de Morais, J.W. 2010. Resource distribution and soil moisture content can regulate bait control in an ant assemblage in Central Amazonian forest. Austral Ecology, 35: 274-281.

Benson, W.; Harada, A.Y. 1988. Local diversity of tropical and temperature ant faunas (Hymenoptera: Formicidae). Acta Amazonica, 18: 275-289.

Bernstein, R.A. 1979. Schedules of foraging activity in species of ants. Journal of Animal Ecology, 48: 921-930.

Bestelmeyer, B.T. 2000. The trade-off between thermal tolerance and behavioural dominance in a subtropical South American ant community. Journal of Animal Ecology, 69: 998-1009.
Bestelmeyer, B.T.; Agosti, D.; Alonso, L.E.; Brandão, C.R.F.; Brown, J.W.L.; Delabie, J.H. C.; Silvestre, R.; Majer, J.D.; Schultz, T.R. 2000. Field techniques for the study of groud-dwelling ants: an overview, description, and evaluation, p. 122-145. In: Agosti, D.; Majer, J. D.; Alonso, L. E.; Schultz, T. R. (Eds.). Ants: Standard methods for measuring and monitoring biodiversity. Smithsonian Institution Press, Washington.

Brandão, C.R.F.; Silvestre, R.; Reis-Menezes, A. 2000. Influência das interaçóes comportamentais entre espécies de formigas em levantamentos faunísticos em comunidades de cerrado. Oecologia Brasiliensis, 8: 371-403.

Byrne, M. M. 1994. Ecology of twig-dwelling ants in a wet low land tropical forest. Biotropica, 26: 61-72.

Cerdá, X.; Dahbi, A.; Retana, J. 2002. Spatial patterns, temporal variability, and the role of multi-nest colonies in a monogynous Spanish desert ant. Ecological Entomology, 27: 7-15.

Cerdá, X.; Retana, J.; Cros, S. 1998. Critical thermal limits in Mediterranean ant species: trade-off between mortality risk and foraging performace. Functional Ecology, 12: 45-55.

Colwell, R.K.; Coddington, J. 1994. Estimating terrestrial biodiversity through extrapolation. Philosophical Transactions of the Royal Society of London Series B-Biological Sciences, 345: 101-118.

Davidson, D.W. 1997. The role of resource imbalances in the evolutionary ecology of tropical arboreal ants. Biological Journal of the Linnean Society, 23: 153-181.

Davidson, D.W. 1998. Resource discovery versus resource domination in ants: a functional mechanism for breaking the trade-off. Ecological Entomology, 23:484-490.

Dunn, R.R.; Agosti, D.; Andersen, A.N.; Arnan, X.; Bruhl, C.A.; Cerdá, X.; Ellison, A.M.; Fisher, B.L.; Fitzpatrick, M.C.; Gibb, H.; Gotelli, N.J.; Gove, A.D.; Guenard, B.; Janda, M.; Kaspari, M.; Laurent, E.J.; Lessard, J.P.; Longino, J.T.; Majer J.D.; Menke, S.B.; McGlynn, T.P.; Parr, C.L.; Philpott, S.M.; Pfeiffer, M.; Retana, J.; Suarez, A.V.; Vasconcelos, H.L.; Weiser, M.D.; Sanders, N.J. 2009. Climatic drivers of hemispheric asymmetry in global patterns of ant species richness. Ecology letters, 12: 324-33.

Ellwood, M.D.F.; Foster, W.A. 2004. Doubling the estimate of invertebrate biomass in a rainforest canopy. Nature, 429: 549551.

Erwin, T. 1989. Sorting tropical forest canopy samples. Insect Collection News, 2: 8-18.

Feener, Jr. D.H.; Schupp E. W. 1998. Effect of treefall gaps on the patchiness and species richness of Neotropical ant assemblages. Oecologia, 116: 191-201.

Fittkau, E.J.; Klinge, H. 1973. On biomass and trophic structure of the central Amazonian rain forest ecosystem. Biotropica, 5: 2-14.

Gordon, D.M.; Kulig, A. 1996. Founding, foraging, and fighting: colony size and the spatial distribution of harvester ant nests. Ecology, 77: 2393-2409.

Gotelli, N.J.; Colwell, R.K. 2001. Quantifying biodiversity: procedures and pitfalls in the measurement and comparison of species richness. Ecology Letters, 4: 379-391. 
Hahn, D.A.; Wheeler, D.E. 2002. Seasonal Foraging Activity and Bait Preferences of Ants on Barro Colorado Island, Panama. Biotropica, 34: 348-356.

Heard, S.B. 1998. Resource Patch Density and Larval Aggregation in Mushroom-Breeding Flies. Oikos, 81: 187-195.

Holdridge, L.R. 1967. Life zone ecology. Revised edition. Tropical Science Center, San José, Costa Rica. 149 pp.

Holway, D.A.; Lach, L.; Suarez, A.V.; Tsutsui, N.D.; Case, T J. 2002. The causes and consequences of ant invasions. Annual Review of Ecology and Systematics, 33: 181-233.

Kaspari, M.; Yanoviak, S.P. 2001. Bait Use in Tropical Litter and Canopy Ants - Evidence of Differences in Nutrient Limitation. Biotropica, 33:207-211.

Le Breton, J.; Jourdan, H.; Chazeau, J.; Orivel, J.; Dejean, A. 2005. Niche opportunity and ant invasion: the case of Wasmannia auropunctata in a New Caledonian rain forest. Journal of Tropical Ecology, 21: 93-98.

Longino, J.T. 2003. The Crematogaster (Hymenoptera, Formicidae, Myrmicinae) of Costa Rica. Zootaxa, 151: 1-150.

Lovejoy, T.E., Bierregard, R.O.; Gentry, A.H. 1991. Central Amazonian forests and the minimal critical size of ecosystem project, p. 60-71. In: Gentry, A.H. (Ed.) Four Neotropical Rainforests. Yale University Press, New Haven.

Messina, F.J. 1991. Life-history variation in a seed beetle: adult egg-laying vs. larval competitive ability. Oecologia, 85: 447-455.

New, T.R. 2008. Legislative inconsistencies and species conservation status: understanding or confusion? The case of Riekoperla darlingtoni (Plecoptera) in Australia. Journal of Insect Conservation, 12: $1-2$.

Oliver, I.; Beattie, A.J. 1993. A Possible Method for the Rapid Assessment of Biodiversity. Conservation Biology, 7: 562-568.

Oliver, I.; Beattie, A.J. 1996. Designing a cost-effective invertebrate survey: a test of methods for rapid assessment of biodiversity. Ecological Applications, 6: 594-607.

Porter, S.D.; Savignano, D.A. 1990. Invasion of polygyne fire ants decimates native ants disrupts arthropod community. Ecology, 71: 2095-2106.

R Development Core Team 2009. R: A language and environment for statistical computing. R Foundation for Statistical Computing, Vienna, Austria. ISBN 3-900051-07-0, URL http://www.Rproject.org.

Ribeiro, M.N.G.; Adis, J. 1984. Local rainfall variability - A potential bias for bioecological studies in the Central Amazon. Acta Amazonica, 14: 159-174.

Rueda-Almonacid, J.V.; Carr, J.L.; Mittermeier, J.V.; RodríguezMahecha, J.V.; Mast, R.B.; Vogt, R.C.; Rhodin, A.G.J.; OssaVelásquez, J.D.L. O.; Rueda, J.N.; Mittermeier C.G. 2007. Las tortugas y los cocodrilianos de los países andinos Del trópico. Conservación Internacional, Bogotá, Colômbia. 537 pp.

Salati, E. 1985. The climatology and hydrology of Amazonia, p. 1848. In: Prance, G.T.; Lovejoy, T.E. (Eds.). Amazonia. Oxford, Pergamon Press.
Sanders, N.J.; Gordon, D.M. 2002. Resources and the flexible allocation of work in the desert ant, Aphaenogaster cockerelli. Insectes Sociaux, 49: 371-79.

Sanders, N.J.; Gordon, D.M. 2003. Resource-dependent interactions and the organization of desert ant communities. Ecology, 84: 1024-1031.

Savolainen, R.; Vepsäläinen, K. 1988. A competition hierarchy among boreal ants: impact on resource partitioning and community structure. Oikos, 51:135-155.

Silva, R.R.D.; Brandão, C.R.F.; Silvestre, R. 2004. Similarity Between Cerrado Localities in Central and Southeastern Brazil Based on the Dry Season Bait Visitors Ant Fauna. Studies on Neotropical Fauna and Environment, 39: 191-199.

Souza, J.L.P. 2009. Avaliação do esforço amostral, captura de padrốes ecológicos e utilizaçáo de taxa substituto em formigas (Hymenoptera, Formicidae) de serrapilheira com tres métodos de coleta na floresta amazonica, Brasil. Dissertaçáo de Doutorado, Instituto Nacional de Pesquisas da Amazônia, Manaus, Amazonas. 97 pp (in Portuguese)

Stork, N.E. 1991. The composition of the arthropod fauna of Borneo lowland rain forest trees. Journal of Tropical Ecology, 7: 161-180.

Ugland, K.I.; Gray, J.S.; Ellingsen, K.E. 2003. The speciesaccumulation curve and estimation of species richness. Journal of Animal Ecology, 72: 888-897.

Vasconcelos, H.L.; Vilhena, J.M.S. 2006. Species Turnover and Vertical Partitioning of Ant Assemblages in the Brazilian Amazon: A Comparison of Forests and Savannas. Biotropica, 38: 100-106.

Vasconcelos, H.L.; Macedo, A.C.C.; Vilhena, J.M.S. 2003. Influence of topography on the distribution of ground-dwelling ants in an Amazonian forest. Studies on Neotropical Fauna and Environment, 38: $115-124$.

Vasconcelos, H.L.; Vilhena, J.M.S.; Facure, K.G.; Albernaz, A.L.K.M. 2010. Patterns of ant species diversity and turnover across $2000 \mathrm{~km}$ of Amazonian floodplain forest. Journal of Biogeography, 37: 432-440.

Vasconcelos, H.L.; Fraga, N.J; Vilhena, J.M.S. 2004. Formigas do Parque Nacional do Jaú: uma primeira análise, p. 154-160. In: Borges, S.H.; Iwanaga, S,; Durigan, C.C.; Pinheiro, M.R. (Eds.). Janelas para a Biodiversidade no Parque Nacional do Jaú: uma estratégia para o estudo da biodiversidade na Amazônia. Vitória Amazônica, Manaus.

Vepsäläinen, K.; Savolainen, R. 1990. The effect of interference by formicine ants on the foraging of Myrmica. Journal of Animal Ecology, 59: 643-654.

Ward, D.F.; New, T.R.; Yen, A.L. 2001. Effects of pitfall trap spacing on the abundance, richness and composition of invertebrate catches. Journal of Insect Conservation, 5: 47-53.

Yanoviak, S.P.; Kaspari, M. 2000. Community structure and the habitat templet: ants in the tropical forest canopy and litter. Oikos, 89: 259-266.

Recebido em 19/03/2010

Aceito em 06/06/2010 